EDITORIAL

\title{
Depression, stress, and coronary heart disease: the need for more complex models
}

\author{
A Steptoe, D L Whitehead
}

Depression has been related both to the development of
coronary heart disease and to prognosis in patients
following acute myocardial infarction, but the clinical
significance of these associations remains uncertain

(n.m.

$\mathrm{T}$ here is a growing literature relating psychosocial factors with the development of coronary heart disease (CHD), and with prognosis in the patients following acute coronary events. Epidemiological studies, involving prospective designs and concurrent measurement of standard cardiovascular risk factors and lifestyle variables, have identified independent associations between the development of CHD in previously healthy adults and work stress, social isolation, and other forms of chronic stress. ${ }^{1}$ On the psychological side, depression has dominated the literature over recent years. Some 22 longitudinal population studies have been conducted, of which 14 showed depression to be a moderate or strong predictor of future coronary events. ${ }^{1}$

The study by Wolff and colleagues ${ }^{2}$ reported in this issue raises the question of whether depressive symptoms have a special relevance to coronary outcomes beyond the effect of general psychological strain and linked psychosocial factors. They carried out a cross sectional population study with more than 2000 middle aged men and women from north eastern Germany, and assessed psychological strain with a mixed measure including symptoms of anxiety, depression, fatigue, and sleep disturbance. One of the criticisms of psychosocial studies of CHD is that investigators have included "soft" measures such as self reported angina among cardiac outcomes, so that associations between psychological factors and CHD might be caused by subjective reporting biases. ${ }^{3}$ Wolff and colleagues $^{2}$ avoided this problem by assessing carotid atherosclerosis as an objective marker of subclinical disease. They found the expected association between standard risk factors such as hypertension, diabetes, smoking, cholesterol values, and carotid atherosclerosis. In addition, scores on the psychological strain measure were linearly associated with the likelihood of carotid plaque, independently of other factors. These are cross sectional results on a proxy measure of coronary atherosclerosis, so causal conclusions cannot be drawn. Nevertheless, the findings endorse the need to look beyond depression at anxiety and other symptoms of psychological distress. Psychosocial factors such as social
Heart 2005;91:419-420. doi: 10.1136/hrt.2004.045310

isolation and life stress were not assessed, so we do not know whether the psychological strain reported in this population was an intrinsic personality characteristic, or a response to exposure to psychosocial adversity.

Wolff and colleagues ${ }^{2}$ observed no association between psychological strain and carotid intimamedial thickness, and raise the intriguing possibility that plaque development is more closely related to haemostatic and prothrombotic factors than is intima-medial thickening. An influence of psychological experience on these processes is supported by recent evidence that prothrombotic responses are elicited by acute stress, and that these responses are more sustained in healthy adults with elevated psychosocial risk factors, ${ }^{4}$ and in patients with coronary artery disease. ${ }^{5}$

\section{DEPRESSION AND ACUTE CARDIAC EVENTS}

Depression is a common problem in patients following acute cardiac events. It is estimated that up to $20 \%$ of individuals have a major depressive episode within a few weeks of acute events, with a further $25 \%$ experiencing minor depression or dysthymia. Depressed patients experience more social problems over the first year of recovery, report impaired quality of life, are less adherent to treatment advice, and are slower to return to work than non-depressed patients. It is generally agreed therefore that this depression merits treatment in its own right. But debate continues about whether it is a risk factor for future cardiac mortality and morbidity. Several studies have demonstrated that depression following an acute myocardial infarction is associated with increased risk of future cardiac events and cardiac mortality, with adjusted odd ratios of 2 to $3 .{ }^{6}$ However, a number of studies have not shown this relation..$^{-11}$ These discrepancies have highlighted the importance of design, methodological, and statistical issues. Of crucial importance is sample size. ${ }^{6}$ Changes in the management and definition of acute coronary syndromes have led to relatively low mortality rates of between $4-6 \%$ over 12 months in recent studies. ${ }^{12}{ }^{13}$ In order to show a relative risk of mortality of 2.5 in the presence of depression (with a $20 \%$ prevalence), a sample size of more than 700 patients is required $(80 \%$ power, $\mathrm{p}<0.05)$. Several studies that investigated associations with mortality have been underpowered..$^{8} 91113$

Timing of assessment is another important issue. Those studies which have found an effect for depression typically assessed levels during the week or fortnight following admission, while no 
association was observed in an investigation that measured depression after five months. ${ }^{10}$ The context of depressive episodes several months after cardiac events may be quite different from that operating acutely. Levels of depression fluctuate in the months following acute events, and may interact with other psychosocial factors such as social support or marital stress which also change over time and may be affected by the onset of the patient's illness. ${ }^{14} \mathrm{~A}$ third problem is the method of measurement. Both clinical interview and questionnaire measures have been utilised, but among the questionnaire measures, stronger associations have been found for the Beck depression inventory (BDI) than for measures such as the hospital anxiety and depression scale, ${ }^{8}$ the general health questionnaire, ${ }^{10}$ and non-validated measures. ${ }^{7}$

\section{PROBLEMS OF CONFOUNDING}

An additional question that has arisen is not whether depression is a predictor of cardiac mortality following coronary events, but whether it is an independent predictor. ${ }^{15}$ In this issue of the journal, Lane and colleagues ${ }^{16}$ argue that depression may be confounded with disease severity, and that the independent associations with cardiac mortality and morbidity reported by others are due to insufficient statistical control for conventional predictors of outcome. Earlier results from their own study did not provide strong support for this argument, since they did not observe an association between depression and subsequent cardiac mortality or morbidity either before or after taking clinical measures into account. ${ }^{9}$ The present article describes a secondary analysis of this dataset, identifying which clinical factors predicted cardiac or all case mortality at four, 12, and 36 months following acute myocardial infarction. In multivariate analysis, only the presence of heart failure, the prescription of warfarin at discharge, and a combined severity index consisting of these factors together with scores on the Peel index and length of hospital stay predicted mortality. These findings are broadly in line with predictors of larger studies. ${ }^{17}$

Interestingly, the prescription of warfarin on discharge was associated with increased rather than reduced mortality, and it is suggested that this may reflect warfarin being prescribed to sicker patients at higher risk. Information about the precise indicators for prescription is not presented, so it is not clear whether higher death rates were limited to patients with atrial fibrillation, thromboembolism, severe cardiac dysfunction, or were secondary to adverse bleeding events. Depression was not related either to mortality or with these clinical predictors of mortality. The analysis therefore offers no direct evidence that confounding with clinical severity accounts for the associations between depression and mortality observed by others, but cautions researchers to add to their lists of covariates.

The problem of identifying possible confounds of depression and its association with measures of disease and clinical factors is likely to continue. The management of myocardial infarction and even the definitions of acute coronary syndromes are constantly evolving, so it is inevitable that earlier studies will not have the range of indicators that is now thought to be relevant. For example, almost all of the studies of depression in patients following acute myocardial infarction were carried out before prompt revascularisation was introduced into the management repertoire, and pharmacological treatments were also different. The prognostic significance of identifying acute depression will almost certainly change as clinical management is refined. What Lane and colleagues ${ }^{16}$ highlight is the importance in studying depression and CHD to develop a clear model of how the association might be mediated. If depression does affect outcome, it is not a magic ingredient, but must act through pathological processes. There is evidence that depression is associated with inflammation, disturbed autonomic balance, poor adherence to medication and lifestyle advice, and to heart failure. ${ }^{6}$ Taking all factors into account statistically may result in overadjustment, and a loss of information about the processes through which depression might operate.

\section{ACKNOWLEDGEMENTS}

Andrew Steptoe and Daisy Whitehead are supported by the British Heart Foundation.

\section{Authors' affiliations}

A Steptoe, D L Whitehead, Department of Epidemiology and Public Health, University College London, London, UK

\section{REFERENCES}

1 Hemingway $\mathbf{H}$, Kuper $\mathrm{H}$, Marmot M. Psychosocial factors in the primary and secondary prevention of coronary heart disease: an updated systematic review of prospective cohort studies. In: Yusuf S, Cairns JA, Camm AJ, et al, eds. Evidence-based cardiology, 2nd ed. London: BMJ Books, 2003:181-218.

2 Wolff B, Grabe HJ, Völzke H, et al. Relation between psychological strain and carotid atherosclerosis in a general population. Heart 2005;91:460-4.

3 Macleod J, Davey Smith G, Heslop P, et al. Psychological stress and cardiovascular disease: empirical demonstration of bias in a prospective observational study of Scottish men. BMJ 2002;324:1247-51.

4 Steptoe A, Kunz-Ebrecht S, Rumley A, et al. Prolonged elevations in haemostatic and rheological responses following psychological stress in low socioeconomic status men and women. Thromb Haemost 2003;89:83-90.

5 Strike PC, Magid K, Brydon L, et al. Exaggerated platelet and hemodynamic reactivity to mental stress in men with coronary artery disease. Psychosom Med 2004;66:492-500.

6 Lett HS, Blumenthal JA, Babyak MA, et al. Depression as a risk factor for coronary artery disease: evidence, mechanisms, and treatment. Psychosom Med 2004;66:305-15.

7 Jenkinson CM, Madeley RJ, Mitchell JR, et al. The influence of psychosocial factors on survival after myocardial infarction. Public Health 1993;107:305-17.

8 Mayou RA, Gill D, Thompson DR, et al. Depression and anxiety as predictors of outcome after myocardial infarction. Psychosom Med 2000;62:212-9.

9 Lane D, Carroll D, Ring C, et al. Mortality and quality of life 12 months after myocardial infarction: effects of depression and anxiety. Psychosom Med $2001 ; 63: 221-30$

10 Stewart RA, North FM, West TM, et al. Depression and cardiovascular morbidity and mortality: cause or consequence? Eur Heart J 2003;24:2027-37.

11 Strik JJ, Denollet J, Lousberg R, et al. Comparing symptoms of depression and anxiety as predictors of cardiac events and increased health care consumption after myocardial infarction. J Am Coll Cardiol 2003;42:1801-7.

12 Dickens CM, McGowan L, Percival C, et al. Lack of a close confidant, but not depression, predicts further cardiac events after myocardial infarction. Heart 2004;90:518-22.

13 Steeds RP, Bickerton D, Smith MJ, et al. Assessment of depression following acute myocardial infarction using the Beck depression inventory. Heart 2004;90:217-8.

14 Frasure-Smith N, Lesperance F, Gravel G, et al. Social support, depression, and mortality during the first year after myocardial infarction. Circulation 2000;101:1919-24.

15 Lane D, Carroll D, Lip GY. Anxiety, depression, and prognosis after myocardial infarction: is there a causal association? J Am Coll Cardiol 2003;42:1808-10.

16 Lane D, Ring C, Lip GYH, et al. Depression, indirect clinical markers of cardiac disease severity, and mortality following myocardial infarction. Heart 2005;91:531-2.

17 Fox KA. Management of acute coronary syndromes: an update. Heart 2004;90:698-706 